\title{
UNIVERSIDAD DEL CAPITAL HUMANO: DESPLAZAMIENTOS NEOLIBERALES DENTRO DE LA EDUCACIÓN ${ }^{1}$
}

\begin{abstract}
Oscar Espinel ${ }^{2}$
Oscar Pulido ${ }^{3}$

Resumen:

El texto presenta resultados de investigación vinculados a los recorridos históricoepistemológicos del neoliberalismo. El posicionamiento del neoliberalismo como ethos contemporáneo ha producido una época de profundos desplazamientos marcados, principalmente, por el tránsito de la producción material a la producción inmaterial, el auge del capital cognitivo y la expansión global de la economía de mercado. Estas situaciones han transformado la forma universidad y sus posibilidades educativas en la constitución de sujetos y profesionales que respondan a los intereses de este tipo de gubernamentalidad contemporánea. El artículo asume una perspectiva genealógica que se pregunta por el presente de la universidad, sus relaciones con el conocimiento y su función en medio de las sociedades informatizadas. El recorrido permite evidenciar que la incorporación de enunciados como competitividad, innovación y calidad como mecanismos de "modernización" de la universidad, ha generado la reconfiguración de sus fines, escenarios formativos y compromisos con la sociedad y con la cultura.
\end{abstract}

PAlABRAS ClAVE: Universidad. Neoliberalismo. Capital humano. Empresario de sí. Gubernamentalidad.

\section{UNIVERSITY OF HUMAN CAPITAL: NEOLIBERAL DISPLACEMENTS WITHIN EDUCATION}

\begin{abstract}
:
The text presents research results related to the historical-epistemological paths of neoliberalism. The positioning of neoliberalism as a contemporary ethos has produced an era of profound displacements marked mainly by the transition from material to immaterial production, the rise of cognitive capital, and the global expansion of the market economy. These situations have transformed the form university and its educational possibilities in the constitution of subjects and professionals that respond to the interests of this type of contemporary governmentality. The article adopts a genealogical perspective that questions the present of the university, its relationship with knowledge, and its function in the context of computerized societies. This study shows that the incorporation of concepts such as competitiveness, innovation, and quality as mechanisms for the "modernization" of the university has led to the reconfiguration of its objectives, its educational scenarios, and its commitments to society and culture.
\end{abstract}

KEYWORDS: university, neoliberalism, human capital, self-entrepreneur, governmentality

\section{Introducción}

Los cambios geopolíticos, económicos y tecnológicos derivados de la segunda guerra mundial incidieron considerablemente en la concepción del mundo y de

\footnotetext{
${ }^{1}$ Este texto se deriva de los hallazgos y elaboraciones conceptuales del Proyecto Interinstitucional Balance de las formas de enseñanza de la filosofía en Colombia. Entre práctica y experiencia C117-40-160, financiado Dirección General de Investigaciones de la Vicerrectoría General Académica de UNIMINUTO a través de la VII Convocatoria para el desarrollo y fortalecimiento de la investigación en UNIMINUTO y por la Vicerrectoría de Investigaciones de la UPTC.

${ }^{2}$ Doctor en Filosofía. Profesor del Departamento de Filosofía de la Corporación Universitaria Minuto de Dios. Bogotá - Colombia. Grupo de Investigación Pensamiento, Filosofía y Sociedad. oscar.espinel@yahoo.com.

${ }^{3}$ Doctor en Ciencias de la Educación. Profesor de la Escuela de Filosofía de la Universidad Pedagógica y Tecnológica de Colombia (UPTC). Grupo de Investigación Filosofía, Sociedad y Educación (Gifse). oscar.pulido@uptc.edu.co
} 
la sociedad. Como resonancia de estos años convulsos se proyectaron nuevas maneras de direccionar a la sociedad acordes con las dinámicas del mercado; es así como la educación $-\mathrm{y}$, con ella, la universidad que tradicionalmente estuvo alejada de la sociedad—, se convierte en un proyecto de orden mundial dispuesto a transformar la realidad del sujeto con el fin de ponerlo en capacidad de responder a estas nuevas sociedades que empiezan a abrirse campo. En efecto, la educación se convirtió en mecanismo sutil y bastante efectivo de producción de subjetividades para responder a las dinámicas y demandas más recientes. ${ }^{4}$ De acuerdo con las recomendaciones de las misiones internacionales (dirigidas por la UNESCO, CEPAL, Banco Mundial, Banco Interamericano de Desarrollo, entre otros) cada vez se fue haciendo más urgente modernizar la educación y, en consecuencia, a la universidad. En efecto, la universidad fue objeto de una transformación radical y estructural profunda, ya no con una perspectiva cultural que respondiera a los intereses de las élites, sino con el objeto de hacerla productiva como estrategia para que la sociedad pudiese ponerse en sintonía con el modelo económico imperante. ¿Qué efectos produjo la connotada modernización en el escenario educativo? ¿Cuál es entonces el lugar y función que asume la educación en el mundo contemporáneo? ¿Qué es el neoliberalismo y qué efectos produce en la educación y la universidad?

El análisis asume una perspectiva genealógica que, a partir de los cuestionamientos anteriores, rastrea en discursos y prácticas de mediados del siglo XX las posibles superficies de emergencia de las transformaciones de la universidad en los años 80 y 90 del siglo XX y comienzos del siglo XXI. De esta manera, se apropian conceptos de Michel Foucault que, a manera de categorías teórico-metodológicas, se emplean para leer el neoliberalismo en su aparición histórica. Es de resaltar la capacidad de estas categorías foucaultianas para propiciar una mirada panorámica de un problema $\mathrm{y}$, en nuestro caso, de una forma-problema que atraviesa las preocupaciones educativas y sociales en torno a la universidad en Colombia.

El texto se organiza en cuatro (4) apartados centrales para luego cerrar con algunas conclusiones generales. El primero de ellos hace referencia a las relaciones entre el capitalismo en su fase de neoliberalismo y la consolidación de los sistemas educativos, en especial, de la educación superior y la universidad; luego, en un segundo momento, se

\footnotetext{
${ }^{4}$ Así lo dejan ver, por ejemplo, Militão, Santana y Perboni en su artículo La expansión de los sistemas evaluadores y la teoría crítica: un diálogo (in) pertinente: "A sociedade contemporânea apesenta-se marcada pela tecnologia, pela fluidez e pela transitoriedade dos conhecimentos. Nesse mosaico construído cotidianamente pelo homem, poucas são as certezas nas quais podemos crer, e uma delas chama muita atenção: a educação" (2019, p. 2048).
}

\begin{tabular}{|l|l|l|l|l|}
\hline Qonista Zialectus & Ano 10 & n. 22 & Edição Especial, junho 2021 & p. 26- 41 \\
\hline
\end{tabular}


concentra en la descripción de la gubernamentalidad neoliberal tanto desde sus efectos como de sus apropiaciones; en tercer lugar, aborda la compresión del valor económico de la educación en los economistas neoliberales y su dispersión discursiva, en especial, en las territorialidades de la universidad colombiana. Para finalizar, se describe el capital humano como la dimensión educativa del neoliberalismo, que recurre a las posibilidades del aprendizaje como ejercicio de individuación y de autoexplotación contemporánea.

\section{Capitalismo y sistema educativo}

A lo largo de las últimas décadas el capitalismo ha entrado en una profunda revolución financiera, tecnológica y cultural que ha generado la emergencia de nuevas prácticas y maneras de acumulación del capital. Esta serie de transformaciones han sido estudiadas y nombradas desde distintos tópicos como sociedad posindustrial, sociedad poscapitalista, era posmoderna, sociedad de control, capitalismo cognitivo e, incluso, en un guiño más próximo al campo educativo, como sociedad del aprendizaje (NOGUERA, 2012). Al mismo tiempo, una de sus manifestaciones más expandidas coincide con aquello que, de manera general, se identifica como neoliberalismo el cual, más que un modelo de acción social y económica se encumbra como una manera de ser y de producir subjetividad(es).

Tal como lo muestra Daniel Cohen (2007), se produce el paso de una sociedad centrada en la producción masiva hacia una sociedad estructurada a partir de los "servicios". La venta y prestación de "servicios" desplaza la otrora centralidad de la fuerza de trabajo, de la misma manera que el mercado desplaza la centralidad de la producción en masa, características vertebrales de las sociedades derivadas de la revolución industrial europea y su expansión. Esto puede traducirse, siguiendo a Cohen (2007, p 13), en el hecho de que, dentro de las sociedades contemporáneas, "la materia trabajada por el hombre es el propio hombre”, superando así la relación feudal — colonial en territorio americano- con la tierra, al igual que el lugar central que ocupaba la manufactura de materia prima en la gran fábrica del siglo XIX y comienzos del XX. La producción material cede ante el ímpetu de la producción inmaterial y con ello emerge la posibilidad de una empresa sin trabajadores, de un empleado que hace de sí mismo su propia empresa y de formas de trabajo desterritorializadas y atemporales, flexibles y permanentes. Virtualización tanto de espacialidades como de subjetividades y productos. De hecho, la misma moneda de intercambio logra índices extraordinarios de virtualización bajo la expansión financiera a cada rincón de la vida (bancarización, bolsa de valores, sistemas de crédito, dinero virtual, plataformas digitales, internetización del

\begin{tabular}{|l|l|l|l|l|}
\hline Revista Dialectus & Ano 10 & n. 22 & Edição Especial, junho 2021 & p. 26- 41
\end{tabular}


mercado, etc.). Las transacciones financieras y mercantiles traducidas a operaciones algorítmicas transforman radicalmente la circulación del capital y su poder adquisitivo. Asimismo, el dinero, el mercado de créditos y seguros, los intereses sobre la deuda y la especulación se transforman en la principal mercancía del boyante capital financiero de carácter global.

De otra parte, el neoliberalismo, como "estilo de vida" (ethos) contemporáneo, no solo reconfigura las relaciones económicas y laborales, sino que en su apogeo transforma las formas habituales de ser y hacer; produce subjetividades singulares propias de la expansión de un modelo como el de la empresa, incluso, a los confines mismos de los procesos de subjetivación. El modelo empresa termina por permear cada espacio de la vida contemporánea y convierte al sujeto en un empresario de sí. De modo que, la expansión del modelo empresa significará la reconfiguración de las formas de gobierno sobre la vida.

Al lado de una sociedad con características de servicios en donde el hombre es objeto y sujeto del consumo - así como de la transacción-, aparece otra característica no menos importante: la centralidad del conocimiento y la información. Centralidad destacada incluso en el nombre que recibe dicha sociedad: "Sociedad de la Información" o "Sociedad del Conocimiento". En medio de este horizonte, la educación y su forma universidad, se hacen objeto de una transformación radical y estructural profunda, pues luego de llegar a constituirse durante siglos y a lo largo de todo occidente, como un bien cultural - usualmente - exclusivo para reducidas capas sociales, muta para convertirse en un bien económico para los individuos y, desde allí, para la sociedad en general. Lo cual implica una especie de democratización del conocimiento que complementa la universalización de la escolarización iniciada con los Sistemas de Instrucción Pública en el transcurso del largo siglo XIX. Movimiento que se vincula a la inversión de las relaciones entre la economía y la política.

Durante largo tiempo, la creación de la riqueza fue asunto preponderante del Estado, eje de su crecimiento como aparato soberano y objeto de extrema regulación estatal; no obstante, en tiempos más recientes, la relación parece dar un giro toda vez que la economía, de manera paulatina pero decisiva, será quien rija y ordene el mundo social, cultural y político. Lo cual significa el posicionamiento de un Estado sistemáticamente puesto al servicio del mercado.

El informe presentado por Philip Coombs (1915-2006) por encargo de la UNESCO se presenta como eximia superficie de emergencia de la transfiguración que

\begin{tabular}{|c|c|c|c|}
\hline Rovista Dialeatus & Ano 10 & n. 22 & Edição Especial, junho 2021 \\
\hline
\end{tabular}


venimos rastreando en el seno de la educación. El estudio presentado en 1967 por el economista estadounidense se encarga de diagnosticar cierto anacronismo de la educación en relación con las demandas del mercado mundial. De suerte que, en estricto sentido, la "crisis" diagnosticada responde al desfase de los sistemas educativos del planeta de cara a las dinámicas económicas en juego desde la segunda mitad el siglo XX. Este es, precisamente, el título que Coombs (1978) asigna a dicho informe: "La crisis mundial de la educación".

Una "crisis" que, por definición, exige tomar medidas y adelantar los ajustes necesarios. Esta vía será la reestructuración de los sistemas educativos nacionales de todo el planeta. En consecuencia, las reformas educativas serán uno de los mecanismos, quizás uno de los más efectivos, para la adecuación de las naciones a las nuevas exigencias del mercado mundial. ${ }^{5}$ Dicho de otro modo, esta invocación de la crisis dentro del análisis de carácter mundial (ya no local, ni nacional exclusivamente) supone, reclama y legitima la articulación de los sistemas educativos locales con las políticas y lineamientos de orden mundial. Lineamientos que, por supuesto, responden a los reacomodamientos del orden económico, también de carácter mundial. Tal es la reestructuración que anticipa Coombs, reconocido economista de la Universidad de Chicago. Aquí cabe recordar que Coombs se desempeñó como secretario de educación del presidente estadounidense Jhon F. Kennedy (1961) y actuó permanentemente como asesor de las reformas educativas emprendidas desde entonces en distintos países del mundo bajo la impronta tutelar de la UNESCO.

La educación es identificada como un factor económico fundamental dentro de la reactivación y crecimiento de las economías locales y regionales. Lo cual transforma la mejora de los sistemas educativos y la formación del denominado "capital humano", en una inversión estratégica dentro del concierto internacional abierto en el periodo de posguerra (SCHULTZ, 1968; BECKER, 1964; 1983). El conocimiento, la educación y, con ellas, la investigación y la innovación, se convertirán en factores preponderantes para la competitividad en el contexto del mercado mundial (LAVAL, 2004; LAVAL; DARDOT, 2013). En consonancia con todas estas tendencias, a partir de la década de

\footnotetext{
${ }^{5}$ Para ampliar estos análisis puede revisarse el exhaustivo estudio de Alberto Martínez-Boom (2004), titulado De la escuela expansiva a la escuela competitiva. Dos modos de modernización en América Latina. Igualmente, el trabajo recogido en La universidad como proyecto modernizador. Ilusiones y desencantos (PULIDO, 2018) aporta elementos para estudiar la transfiguración de la universidad colombiana a partir de la estrategia de las reformas y su envés, la crisis.
} 
1970 y, de manera contundente en la década de $1990,{ }^{6}$ se incorporarán al aparato educativo colombiano lógicas preponderantes de planeación, cuantificación y control en función de enunciados como desempeño, capacidad productiva, calidad, innovación, eficiencia y competitividad.

\section{Gubernamentalidad neoliberal}

Ya en el tránsito entre los siglos XIX y XX, el liberalismo muestra una profunda crisis por cuenta del desarrollo consecuente de sus más caros principios y las más recientes "mutaciones" del capitalismo (LANGASNERIE, 2015). De allí la decadencia o descentramiento de las grandes instituciones sobre las que se construyeron las sociedades y que operaban como ejes gravitacionales de la vida. Nos referimos a instituciones como la familia, la escuela, la Iglesia, la fábrica y el mismo Estado (DELEUZE, 1999). Tránsito desde una sociedad institucionalo-céntrica cuyo núcleo estructural eran instituciones como las citadas, a una desinstitucionalización de las sociedades ante la emergencia de otras formas de organizar la vida. O, mejor, emergencia de otras estructuras institucionalizantes más abiertas y móviles como podría ser el caso, por ejemplo, del mercado.

El neoliberalismo, muy al contrario de lo que se piensa, se enfrenta a una nueva bipolaridad generada por las alianzas estratégicas que atravesaron el siglo XIX y XX. Por un lado, combate el inmovilismo producido por el liberalismo y por otro, la hegemonía del socialismo como modelo transformador frente a un mundo que se deshace ante el crecimiento de la miseria y el hambre. Lo cual significará que, de acuerdo con Lagasnerie, el neoliberalismo se presente como productor de utopía y cambio social en abierta disputa con las referencias socialistas. Ciertamente, el neoliberalismo se asume a sí mismo revolucionario en cuanto promueve una transformación radical de las estructuras sociales, políticas y económicas en oposición al excesivo intervencionismo de la maquinaria estatal liberal y el Estado de bienestar que terminaba por hacer aún más pesado dicho aparato.

\footnotetext{
${ }^{6}$ Por ejemplo, para el caso colombiano, en 1994 se elabora la tercera gran reforma educativa del país en toda su historia, con la formulación de la Ley 115 conocida como Ley General de Educación y, en particular para la educación superior, se expide en 1992 la Ley 30 mediante la cual se sustenta la consecuente reestructuración del sistema educativo en los niveles avanzados. A partir de entonces el sistema educativo experimentará un cambio profundo en sus propósitos, gramática, discursos y estructura. Reforma apoyada en reacomodamientos de orden global como las ya impulsadas por Edgar Faure en 1972 Aprender a ser. La educación del futuro o Jacques Delors en 1994 en el informe para la UNESCO intitulado La educación encierra un tesoro.
}

\begin{tabular}{|l|l|l|l|l|}
\hline Q ovista Dialectus & Ano 10 & n. 22 & Edição Especial, junho 2021 & p. 26-41 \\
\hline
\end{tabular}


Así pues, en el marco de los Estados modernos la crisis del liberalismo se traduce en una crisis de la gubernamentalidad liberal " $O$ sea, una crisis que plantea esencialmente el problema de la intervención política en materia económica y social, así como su justificación doctrinal" (LAVAL; DARDOT, 2013, p.30). La crisis del liberalismo deriva en la crisis de gubernamentalidad que dará entrada a una nueva forma de agenciamiento sobre las poblaciones y la gestión de la vida: la gubernamentalidad neoliberal. Más que la vida biológica y los cuerpos individualizados, el Estado y las nuevas instancias de gobernamiento como el mercado, se dirigirán hacia el alma (noopolítica) y el mundo de los deseos. Más que administrar y controlar se tratará de gestionar, actuar a distancia, disponer para orientar hacia formas de vida autorreguladas y autodireccionadas hacia fines comunes, estilos de vida impuesto por las lógicas de mercado y, en últimas, hacia la "vida de consumo". De este modo, el arte de gobernar se redirige desde la administración de la vida biológica al moldeamiento de los sujetos deseantes.

Transformación radical que desplaza la centralidad de las instituciones tradicionales, la solidez de las estructuras y la estabilidad del orden para propiciar la flexibilidad, la alteración de los tiempos y espacios y el desanclaje de las pesadas instituciones, hábitos y tradiciones. A lo cual se suma la configuración de nuevos sujetos con nuevas características: emprendedores, competitivos y consumidores. Un sujeto neoliberal que abandona el hogar de la nación para hacerse ciudadano del mundo. Frente a la estabilidad, la quietud y las fuerzas gravitacionales del otrora Estado-nación y sus instituciones se posicionan la aceleración, la desterritorialización, la levedad (ingravedad), la flexibilidad, la virtualización como nuevos principios rectores de los ritmos y espacios de la vida. El neoliberalismo es, afirma Foucault, una ética, una forma de vida, un "foco utópico", un "humor", una manera de vivir.

\section{El valor económico de la educación y bien-estar}

De este modo, los cambios y las transformaciones agenciadas por el nuevo capitalismo que se consolida a lo largo de la segunda mitad del siglo XX y que ingresa a una nueva fase de reformulación en la década de 1980 ante lo que autores como MartínezBoom (2004) identifican como el agotamiento del modelo de desarrollo, plantean para la sociedad y para la educación superior, en su forma de Universidad, profundas revisiones en su relación con el conocimiento (PULIDO, 2016). Para muchos sectores de la crítica, la Universidad (en particular para este estudio, la Universidad colombiana) parece estar atrapada por los discursos de gestión, competencias, calidad y, recientemente, por

\begin{tabular}{|l|l|l|l|l|}
\hline Qovista Dialectus & Ano 10 & n. 22 & Edição Especial, junho 2021 & p. 26-41 \\
\hline
\end{tabular}


innovadores modelos como el de capacidades y resultados de aprendizaje. La educación se vuelca al desarrollo de habilidades para la resolución de problemas inmediatos y productivos. Toda una ingeniería curricular puesta al servicio de la producción de conocimiento y el desarrollo de aptitudes para el refinamiento del recurso humano que será articulado a las dinámicas de producción y consumo. ${ }^{7}$

En efecto, el hombre contemporáneo es un sujeto que invierte en sí mismo para alcanzar mayores niveles de competitividad en la era del mercado. Despliega sobre sí, en términos de Sloterdijk (2012), toda una serie de antropotécnicas y formas ejercitantes que lo transforman en lo que Foucault (2010) denominará como empresario de sí. En este sentido, el problema a rastrear en esta exploración gira en torno a las prácticas educativas que son expresiones de la gubernamentalidad neoliberal, en donde el gobierno será ejercido desde y sobre los deseos e intereses. Dicho todo lo anterior, surgen algunas inquietudes en torno a la universidad del siglo XXI ¿De qué universidad estamos hablando? ¿Cómo incide este viraje desde el capital humano, el empresario de sí y la producción inmaterial en la arquitectónica y función de la universidad contemporánea? ¿En qué medida la configuración como empresario de sí, los discursos de competitividad y la concepción de la educación como inversión se vinculan con las formas contemporáneas de la gubernamentalidad neoliberal?

Continuando con este recorrido, es necesario anotar que el concepto de "capital humano" emerge en el seno de las discusiones frente al valor económico de la educación. Theodore W. Schultz (1968), en un texto titulado The Economic Value of Education, plantea ya este creciente interés desde la década de 1960; un interés que, llamativamente, se consolida en el ámbito de la economía y de los diseños políticos, y no tanto en el campo de los educadores y pedagogos. El economista estadounidense considera $-\mathrm{y}$ es su argumento central - que "en el aumento de las capacidades adquiridas de la gente en el mundo entero, y en los adelantos cognoscitivos útiles está la clave de la futura productividad económica y de sus contribuciones al bienestar humano" (SCHULTZ, 1985, p. 9). En este orden de ideas, de acuerdo con Schultz, la productividad se verá incrementada por factores distintos al capital mismo ya que la creación de riqueza será puesta en otro lado; esto es, en el sujeto mismo que hace de sí su propio producto y la más rentable mercancía. Todo ello deriva en una profunda transformación tanto de las

${ }^{7}$ Algunas de estas ideas que aquí retomamos han sido desarrolladas con mayor amplitud en Pulido (2016). 
formas de producción como de las concepciones frente al trabajo, al trabajador y la mercancía a comercializar.

Schultz se enfoca en rastrear la cantidad creciente de personas que invierten grandes recursos en sí mismas y, en particular, en su formación como agentes humanos. Así, continúa precisando Schultz, "los factores de producción decisivos para el mejoramiento del bienestar de los pobres no son el espacio, la energía y la disponibilidad de tierra cultivable. Los factores decisivos son el mejoramiento de la calidad de la población y los adelantos en el conocimiento" (SCHULTZ, 1985, p. 13. Énfasis nuestro). Todo lo cual le autoriza a identificar a la educación como una importante fuente de producción y acumulación de capital.

A partir de sus análisis en torno a las relaciones entre economía y educación, Schultz afirma que la formación no es solo una inversión en el ser humano contemporáneo para la mejora en la calidad de la población, sino que sus efectos asumirán la forma de capital; y, de hecho, uno de los capitales más valiosos y rentables en tiempos actuales. Siguiendo con Schultz, el valor económico de la educación no reside en aquello que históricamente ofrecía, es decir, en la transmisión cultural, la incorporación de las nuevas generaciones a los códigos sociales o los procesos de socialización; por el contrario, desde la perspectiva económica que asume a la educación como problema, la educación se hace valiosa por el retorno económico que sugiere y la dinamización de la producción que implica. Así, con estas características, la educación ingresa a la arena de las políticas públicas y a las estadísticas de las tablas de rendimiento en tanto elemento nuclear dentro del crecimiento económico de cada país.

Ahora bien, el capital humano se forma, se produce y modela de manera preponderante a través de los sistemas educativos, la instrucción y los lineamientos globales en política educativa. La educación se convierte así en un factor económico fundamental, pero también en escenario de subjetivación y estrategia gubernamental, ahora, a escala mundial. Tal como lo demuestran las investigaciones de Schultz (1968) desde la década de 1960, la inversión en capital humano desplegada a partir del aparato escolar y universitario, ha hecho que las tasas de crecimiento económico sean superiores en aquellas naciones en las que ha tenido lugar.

Efectivamente, la creciente especialización de los modelos de producción y de las formas de trabajo, de la mano de los avances tecnológicos, exigirá cada vez mayor especialización de los agentes productivos. Especialización y desarrollo de destrezas que la educación debe suministrar. La ecuación, entonces, se torna sencilla: a mayor

\begin{tabular}{|l|l|l|l|l|}
\hline Rovista Dialectus & Ano 10 & n. 22 & Edição Especial, junho 2021 & p. 26- 41 \\
\hline
\end{tabular}


crecimiento económico y tecnológico, mayor necesidad de educación y, por supuesto, más altos niveles de especialización. Esto ha llevado, en las últimas décadas, a la creciente exigencia de capacitación y actualización permanente.

\section{El capital humano como principio ordenador}

Por su parte, Gary Becker (1930-2014) publica uno de sus trabajos más reconocidos en 1964 y lo titula Human Capital (BECKER, 1964). Becker estudia economía en la Universidad de Chicago de la mano de profesores como Milton Friedman y Alfred Schultz. La teoría del capital humano formula que el caudal de conocimientos de los sujetos, el nivel de destreza de los mismos y la calidad de su formación terminan por determinar (de manera decidida) el grado de productividad de las empresas e instituciones económicas. Lo que significa, a su vez, que el desempeño productivo del trabajador se deriva directamente de la educación recibida, del conjunto de conocimientos adquiridos (dentro y fuera de la empresa) y del desarrollo de los talentos necesarios para la ejecución de actividades específicas. En este sentido, en opinión de Becker (1983), el capital humano opera como parangón del capital físico.

Efectivamente, esta acepción como "capital" permite su acumulación en la medida que se vuelve medible, controlable e intercambiable a través de la inversión, la rentabilidad y las formas de transacción. De esta forma, el capital humano ingresa a la esfera de la oferta y la demanda convirtiéndose en uno de los factores determinantes en el crecimiento económico tanto de las empresas como de las mismas naciones. Y la educación, por su parte, se sitúa como el factor fundamental en la producción y cualificación del capital humano.

Para Becker la inversión en capital humano no se refiere exclusivamente a la educación formal sino que también apunta a los cuidados de orden familiar desde los primeros años de vida. De suerte que la adecuada nutrición, el cuidado para un cuerpo sano, el desarrollo de habilidades básicas tanto motrices como sociales e, incluso, los acumulados culturales que posibilitan nichos sociales más favorables, terminan siendo factores determinantes dentro de la optimización del recurso humano y las condiciones de competitividad. En este orden de ideas, las acciones de la vida familiar se encaminan, de una u otra forma, hacia la garantía del éxito personal en la vida adulta. Tal será el caso de la educación desde la primera infancia, los programas de estimulación temprana y las actividades complementarias a la escolarización.

Ciertamente, en tiempos recientes el cuidado de los padres y las formas de crianza contemporáneas centran la atención en aspectos emocionales y relacionales como

\begin{tabular}{|l|l|l|l|l|}
\hline Qovista Zialectus & Ano 10 & n. 22 & Edição Especial, junho 2021 & p. 26-41 \\
\hline
\end{tabular}


la confianza en sí mismo, la autoestima, la convicción, las habilidades de socialización, la autorregulación emocional, y hasta la capacidad de resiliencia para sobreponerse a las contrariedades y al fracaso. Visto desde esta óptica, la formación de capital humano excede las temporalidades y espacialidades del aparato escolar. En efecto, el desarrollo de las capacidades va de la mano del control de las emociones y su capitalización en los distintos espacios de la intimidad y la vida social. Todas ellas habilidades esenciales dentro del escenario competitivo abierto por la era del mercado neoliberal en la que el producto es, recordémoslo, el mismo hombre.

De otra parte, Becker descubre desde la década de 1960 que, junto a la escuela (en el amplio sentido del término), la empresa también se constituye en escenario privilegiado de formación del capital humano. La experiencia acumulada y el desempeño en las dinámicas de producción representan claramente un importante espacio de formación y capacitación (BECKER, 1964; 1983). Lo cual significa que aquellos trabajadores con mayores condiciones y aptitudes para aprender ascenderán y lograrán mayores niveles de acumulación de capital, tanto humano, cultural y simbólico como material. La clave está en la disposición y capacidad de aprender para luego rentabilizar la experiencia adquirida a través de las diversas y sutiles formas que adquiere el capital. De allí la exigencia de habilidades como aprender a aprender, el aprendizaje a lo largo de la vida y la misma centralidad en el aprendizaje dentro de los aparatos escolares desplazando a la enseñanza. Se trata, en esencia, de adquirir destrezas que permitan capitalizar la experiencia y los aprendizajes puesto que la capacidad de aprender y el trabajo cognitivo serán el capital más valioso para intercambiar en el mercado global.

De este modo, la velocidad y el estar dotado de las herramientas necesarias, tanto cognitivas, intuitivas y emocionales como materiales y tecnológicas, se constituyen en un valor fundamental dentro del aguerrido mundo de la competencia. La cualificación, actualización y preparación para la novedad, así como el acceso a la información, determinarán el nivel de retribución y sus rendimientos. Todo lo cual, como efecto colateral, aumentará los niveles de desigualdad y exclusión en coherencia con el mundo de la competencia que empieza a abrirse paso. En una suerte de darwinismo social, la educación empieza a operar como mecanismo de selección y optimización del recurso humano, mientras el capital cultural y la habilidad para rentabilizar toda experiencia en aprendizaje se transforman en capital (acumulable e intercambiable).

Todo ello permite entender la adecuación de los aparatos escolares — en todos sus niveles formativos - a modelos enfocados en el desarrollo de competencias

\begin{tabular}{|c|c|c|c|}
\hline Rovista Dialeatus & Ano 10 & n. 22 & Edição Especial, junho 2021 \\
\hline
\end{tabular}


orientadas a la optimización de los procesos de adquisición y cualificación de aprendizajes. El objetivo de la educación contemporánea, junto con la preparación para el mundo laboral, girará en torno a la formación de sujetos con la maleabilidad suficiente para adaptarse rápidamente a entornos volubles y, sobre todo, sensibles a las velocidades del mundo actual. Flexibilidad, adaptabilidad, proactividad e iniciativa serán las nuevas virtudes de los sujetos empresarios de sí.

En este orden de ideas, la educación se hará objeto de consumo, rentabilización y producción. La mejora en las capacidades y condiciones para la productividad implicará la posibilidad de aumentar los índices de consumo. Un sujeto productor es, al mismo tiempo, un sujeto consumidor. ${ }^{8}$ La educación no escapa a esta fórmula, es más, termina preparando para ello. Consumir, producir, acumular para volver a consumir, en una espiral interminable. Junto a la centralidad del consumo, reconfigurado como valor en sí mismo y vinculado a la productividad, Becker menciona la aptitud. Cierta disposición en los sujetos que los hace más aptos y, por tanto, mejor equipados para la voracidad de la vida social y económica. La supervivencia del más hábil y mejor dotado que nos hace recordar, una vez más, las amañadas interpretaciones de las teorías darwinistas y su adaptación al mundo socio-económico anticipado por el spencerismo del siglo XIX (LAVAL; DARDOT, 2013, pp. 45-46). ${ }^{9}$ Una aptitud que, además, puede ser nutrida, formada y modelada a partir de mecanismos sociales, culturales y educativos. Lejos de insistir en cierto determinismo biológico, la teoría del capital humano evidencia la capacidad del sujeto de producirse a sí mismo y forjarse como empresario de sí. Aptitudes que, además, no solo pueden crearse y moldearse, sino que también pueden acumularse.

La educación, en otro momento imaginada como derecho y escenario emancipador, paulatinamente, bajo su mercantilización, se va transformando en instrumento de acaparamiento y, por tanto, en instrumento de marginalización, recrudecimiento de los abismos sociales y sometimiento voluntario. Se trata de las formas contemporáneas de gobierno bajo los altisonantes discursos del emprendimiento,

8 A este respecto son bastante ilustrativos los análisis del artículo Inclusão e Aprendizagem como
mperativos da Governamentalidade Neoliberal e a Criação dos Institutos Federais de Educação no Brasil.
En uno de sus apartes oímos decir a las autoras: "A necessidade de capacitar os indivíduos para que passem
a se mover na lógica de aprender, acompanhar as tecnologias, produzir, consumire manter o motor
econômico funcionando implica colocar os indivíduos em movimento, circulando, produzindo,
consumindo mais potentemente" (GATTERMAN; POSSA, 2018, p. 1635).
9 Dice Laval: "aunque el evolucionismo biológico de Spencer les parecerá a ciertos neoliberales muy
anticuado, hasta tal punto que muy a menudo «olvidarán» mencionarlo, salvo para rechazarlo, dejó sin
embargo una marca profunda en el curso ulterior de la doctrina liberal" (LAVAL; DARDOT, 2013, p. 44).

Ano 10

n. 22 Edição Especial, junho 2021

p. $26-41$ 
capacitación, innovación, capital humano, e, incluso, autoexplotación. La educación y la formación de dichas aptitudes para la competencia se trasladan al campo de la responsabilidad individual. En definitiva, vemos emerger tres efectos altamente visibles: individualismo, competitividad y segregación.

\section{ALGUNA NOTAS FINALES}

En la entrevista titulada "Educar ya no significa transmitir y memorizar información" la especialista y, seguramente, líder en innovación educativa, responde a la pregunta "¿Qué habilidades debe adquirir un estudiante para afrontar su vida y desempeñar los trabajos del futuro?" del siguiente modo:

Hace una década, estudiar una carrera y un máster ofrecía una alta probabilidad de conseguir un buen puesto de trabajo y una cierta seguridad económica para realizar unos planes de vida. Ahora prácticamente nadie se atrevería a asegurarle eso mismo a ningún joven en edad de elegir un camino profesional (en empresa privada).

Y es que el nuevo paradigma está exigiendo adaptar los puestos de trabajo: unos desaparecen y otros diferentes surgen. Por esta razón, entrenar las habilidades blandas o 'soft skills', como, por ejemplo, la creatividad, el autoliderazgo, la resiliencia, el pensamiento crítico, la proactividad, la capacidad de aprendizaje, la toma de decisiones o el trabajo en equipo, se ha convertido en una tarea urgente. ${ }^{10}$

La entrevista es publicada en la Revista Digital Educación 3.0 en el mes de marzo de 2019 que, además, se presenta a sí misma como "líder informativo en innovación educativa". Interesa resaltar, por ahora, un par de elementos. Un primer comentario general gira en torno a la individualización y responsabilidad de cada uno frente a la inestabilidad y voracidad de un mercado laboral salvaje y además copado. Un mercado laboral fluctuante y acelerado. Ante la escasez de posibilidades es de suma importancia prepararse muy bien; de lo contrario, se corre el riesgo de perecer. Una vez más, la escasez y la inestabilidad como formas de gobernar. Escasez de ofertas y saturación de demanda que deriva, seguramente, en precarización de las formas de trabajo y rentabilización (especulación) de los escenarios de capacitación.

Es necesario estar preparado para el cambio y adaptarse a las exigencias del mercado. Incluso, es necesario pulir los talentos y enfocarse, de acuerdo con la recomendación de la líder en innovación, en las soft skill en tano plus de la cualificación personal. Es decir, apuntar en una nueva dirección en la que quizás no se había reparado

10 Tomado de https://www.educaciontrespuntocero.com/entrevistas/sara-moraleja-softskills/?fbclid=IwAR2T84-3fa8ovqRQxtspg8Y7zKiWuSO2hxtxTyXA3Ls8Ry1XhhyBVzrtBrU

\begin{tabular}{|l|l|l|l|l|}
\hline Rovista Dialectus & Ano 10 & n. 22 & Edição Especial, junho 2021 & p. 26- 41 \\
\hline
\end{tabular}


lo suficiente Allí radica, justamente, la innovación de la propuesta en cuestión; innovación, además, replicada como estrategia de marketing en medio de la variada oferta educativa con el propósito de ganar mayor impacto en el target poblacional identificado como nicho de mercado.

La especialista citada, termina su respuesta con la siguiente recomendación: "Eduquemos para que los estudiantes desarrollen sus habilidades blandas y descubran sus talentos, de este modo, tendrán más posibilidades para conocerse a sí mismos, ser felices y aportar valor a la sociedad". El objetivo final es crear valor a través de "modelos pedagógicos" innovadores y eficientes para que los sujetos allí formados estén en capacidad, a su vez, de "aportar valor" a la sociedad, a la empresa, al producto, en fin, a lo que sea y en lo que sea. Crear valor y formar para crear valor. Principio fundamental en el tránsito de la producción material a la producción inmaterial de la era neoliberal en la que la felicidad se convierte en otro bien de consumo e instrumento de conducción de las conductas.

Es notable en este ejemplo traído del archivo, el valor económico desde el que se vislumbra y diseña la educación. Racionalidad economicista que adjudica, por extensión, un alto valor de cambio al conocimiento y al aprendizaje. Ciertamente, la herramienta más valiosa que posee el sujeto neoliberal contemporáneo ya no es su fuerza de trabajo sino su inteligencia. Un cerebro productor. Al punto que su mejor capital será la capacidad de producir conocimiento, información y bienes inmateriales. Ello explica, solo por dar un ejemplo más, los desplazamientos de la crítica y su recurrencia altisonante en los modelos "innovadores" de educación tanto escolar como universitaria.

No se trata del pensamiento crítico emancipador del que se hablase hace unas décadas; es decir, el del conocimiento comprometido con la transformación social y el establecimiento de condiciones de vida más justa y dignas para la totalidad de los sectores sociales. ${ }^{11}$ Quizás ello es lo que menos interesa. Se trata, más bien, de la crítica entendida como habilidad. Una destreza en la cual es posible entrenarse y habituarse con el fin de establecer específicas relaciones con el conocimiento. Pero, sobre todo, la habilidad de generar nueva información y alternativas de interpretación. Consumo y producción de información. Producción y consumo de conocimiento. Una manera ligera de aludir al capital cognitivo hacia el que se encaminan y ordenan las instituciones escolares, las prácticas universitarias y la misma investigación.

${ }^{11}$ Algunas indagaciones iniciales respecto a los usos y desplazamientos en torno a la noción de crítica han sido elaboradas en el texto Innovation and critique: passages of a polymorphic relation (ESPINEL, 2020). 
Igualmente se percibe un tránsito desde la enseñanza a la competencia pues, en este sentido, se asiste a la escuela o a la universidad más que para aprender o alcanzar conocimientos, para adquirir habilidades con el fin de hacerse competitivo. Es un asunto de sí consigo. Una tarea de responsabilidad personal. Por ello, junto al pensamiento crítico, también recobra atención la autonomía. Adquirir competencias parece ser la ocupación del sujeto estudiante de la actualidad; competencias y habilidades que, posteriormente, pueden ser ofrecidas en medio de la lógica de oferta y demanda del mercado; incluso, dentro de la misma aula del aparato escolar. Capital acumulable, vendible e intercambiable por otros bienes y servicios. Competencias que empiezan a adquirir un valor creciente en el mercado y que coinciden con la sociedad del aprendizaje, el aprender a aprender y el trabajo intelectual. Cualificación y competitividad que, en los registros del empresario de sí del escenario neoliberal, significa la vigencia y circulación de sí mismo en las redes del mercado global. Sujeto y objeto de consumo, producción e intercambio comercial. Empresario de sí y, simultáneamente, mercancía marcada con crecientes niveles de obsolescencia traducidos en categóricas demandas de actualización y capacitación permanente.

¿De qué universidad estamos hablando? ¿a qué universidad le apuntamos? ¿qué escuela estamos construyendo? ¿qué es y para qué investigar? ¿qué relación establecemos con el conocimiento y qué relación propiciamos en los otros? ¿qué significa ser y hacerse docente? ¿qué significa ser y hacerse estudiante?

\section{REFERENCIAS}

BECKER, Gary. Human Capital. New York: Columbia University Press for the National Bureau of Economic Research, 1964

BECKER, G. El Capital Humano. Un análisis teórico y empírico referido especialmente a la educación. Madrid: Alianza Editorial, 1983

COHEN, Daniel. Tres lecciones sobre la sociedad posindustrial. Buenos Aires: Kats, 2007.

COOMBS, Philip. La crisis mundial de la educación [1968]. Barcelona: Ediciones Península, 1978.

FAURE, Edgar. Aprender a ser. La educación del futuro [1972]. Madrid: Alianza Unesco, 1991.

DELORS, Jacques. La educación encierra un tesoro. Madrid: Santillana - Unesco, 1996 
ESPINEL, Oscar. Innovation and Critique: Passages of a Polymorphic Relation. En: PETERS M.A. y HERAUD R. (Eds.) Encyclopedia of Educational Innovation. Singapore: Springer, 2020. pp. 1-5. https://doi.org/10.1007/978-981-13-2262-4_216-1

FOUCAULT, Michel. Nacimiento de la biopolítica. Buenos Aires: Fondo de Cultura Económica, 2010.

GATTERMANN, Beatris.; POSSA, Leandra Boer. Inclusão e Aprendizagem como Imperativos da Governamentalidade Neoliberal e a Criação dos Institutos Federais de Educação no Brasil. Revista Ibero-Americana de Estudos em Educação, Araraquara, v. 13, n. 4, p. 1632-1651, out./dez., 2018. E-ISSN: 1982-5587. DOI: 10.21723/riaee.unesp.v13.n4.out/dez.2018.11057

LAVAL, Christian. La escuela no es una empresa. Barcelona: Paidós, 2004.

LAVAL, Christian; DARDOT, Pierre. La nueva razón del mundo. Ensayo sobre la sociedad neoliberal. Barcelona: Gedisa, 2013.

MARTÍNEZ-BOOM, Alberto. De la escuela expansiva a la escuela competitiva. Dos modos de modernización en América Latina. Barcelona: Anthropos, 2004.

MILITÃO, Andréia Nunes; SANTANA, Andréia da Cunha Malheiros; PERBONI, Fábio. A expansão dos sistemas avaliativos e a teoria crítica: um diálogo (im) pertinente. Revista Ibero-Americana de Estudos em Educação, Araraquara, v. 14, n. esp. 4, p. 2046-2056, dez., 2019. E-ISSN: 1982-5587. DOI: https://doi.org/10.21723/riaee.v14iesp.4.12927

MINISTERIO DE EDUCACIÓN NACIONAL - MEN. Ley 30 de 1992. Bogotá: MEN, 1992.

MINISTERIO DE EDUCACIÓN NACIONAL - MEN. Ley General de Educación. Bogotá: MEN, 1994

NOGUERA, Carlos. El gobierno pedagógico. Del arte de educar a las tradiciones pedagógicas. Bogotá: Siglo del hombre editores, 2012.

PULIDO, Oscar. Formación de empresarios de sí mismos: miradas a la educación superior en la segunda mitad del siglo XX. En: Espinel, Oscar (Ed.). Pensar a la intemperie. Ensayos filosóficos. Bogotá: Uniminuto, 2016. pp.55-74.

PULIDO, Oscar. La universidad como proyecto modernizador. Ilusiones y desencantos. Tunja: UPTC, 2018.

SCHULTZ, Theodore. Valor económico de la educación. México: UTEHA, 1968.

SCHULTZ, Theodore. Invirtiendo en la gente. Barcelona: Ariel, 1985.

SLOTERDIJK, Peter. Has de cambiar tu vida. Valencia: Pretextos, 2012. 